\title{
Surgery in Safe Hands
}

\author{
Raghvinder Pal Singh Gambhir
}

Published online: 16 November 2010

(C) Association of Surgeons of India 2010

When a medical patient dies the question put to the treating physician is "what happened?" but when a surgical patient dies the question on everyone's lips is "what did you do?"

All of us regularly travel by air and the reputation of the pilot is never a consideration at the time of booking the flight. The name and publicly acknowledged reputation of the surgeon, however, is the subject of much research by the patient and his relatives prior to any surgery, more so in private and non governmental sector. Patients operated in public and teaching hospitals very often do not have the luxury of choice and may have to take the competence of the surgeon for granted. The reasons for this scrutiny are obvious: operation theatres are considered dangerous places. Surgery carried out on the wrong side and retained foreign bodies grab headlines while more common, and often more dangerous, medication or anaesthetic errors get little attention.

There is an inherent risk of human error in any sphere of activity and it is not always due to lack of knowledge or inadequate technical skills. Surgeons are expected to work error-free in an environment where they perform multiple, concurrent tasks, in a setting of high workload, with minimal organisational support. Studies have suggested that over $50 \%$ of harmful effects in patients are preventable $[1,2,3]$. Cost of adverse events, estimated to be greater than $£ 2$ billion in the UK alone, goes beyond the direct costs of medical expenses, and includes loss of income, disability benefits etc. [4]. There is also, loss of trust and confidence in health care providers, therefore something certainly needs to be done.

R. P. S. Gambhir $(\bowtie)$

Armed Forces Medical College,

Pune, India

e-mail: rps_gambhir@rediffmail.com
Conventional approach to failure has been "blame and shame". The culture of blame makes errors something to be concealed, rather than studied, understood and prevented. The mantra today is that "Systems failure" and not individual failure is responsible for adverse events. It is inferred that if an organisation has robust systems in place then errors can be prevented or their effects mitigated. Strategic tools like "root cause analysis" and James Reason's Swiss cheese model have proven very useful in analysing the errors [5]. Airlines, as an industry, were the first to look beyond pilot error and made it mandatory to report any mishap within $24 \mathrm{hrs}$ to avoid punitive action. They also recognised the need for training the crew in nontechnical skills and introduced the crew resource management (CRM) training [6].

Susceptibility to error is an attribute that the medical profession rarely acknowledges and this denial is ingrained in us from medical school and residency. This attitude of personal invulnerability to stress and fatigue has been studied across professions. Consultant surgeons (70\%) are more likely to deny the effect of fatigue on performance than anaesthetists (40\%) or pilots (26\%) [7].

A zero error state is impossible to achieve but there is a considerable room for improvement. In over 234 million surgeries performed annually worldwide, major complications occur in $3-17 \%$, of which over $50 \%$ are avoidable [8]. The incidence of retained sponges or foreign bodies has been estimated to be between 1 in 8,801 to 1 in 18,260 operations [9]. Most of us believe that it will "never happen to me" but $25 \%$ of surgeons are likely to encounter this event at least once or twice in his or her career and a teaching hospital may have to deal with 5-10 such cases annually. The cost to the patient, the hospital and the surgeon can be significant.

Where do we make a start to reduce errors? Standard operating procedures (SOPs) have existed for decades and 
are rarely updated or adhered to fastidiously. Training surgical teams in non-technical skills, communication, briefing and debriefing skills, as well empowering them to be bold so as to raise the red flag or speak up when they see a potential incident or adverse event, are some of the measures that are advocated. Non-technical skills are defined as the cognitive skills (situational awareness, decision making) and social skills (leadership, team work and co-operation) possessed by individuals and demonstrated by a team [4].

WHO recognising the magnitude of the problem, both in developed and developing countries and took up the global patient safety challenge of "safe surgery saves lives" in 2008. One of its initiatives is to propagate the use of surgical safety checklist. The universal adoption of this checklist aims to make operations safer everywhere and anywhere in the world. The 19-item checklist addresses safety issues at three critical points. The first before induction of anaesthesia called the "sign in", then the "time out" before the skin incision and finally the "sign out" after the procedure but before the patient leaves the table. This is an initiative that we all need to support and adopt as it has been shown to be beneficial in reducing adverse events among diverse populations of varied economic circumstances globally $[10,11]$. In a country like India where surgical treatment is provided in most diverse environments, from the five star corporate hospitals to centres in rural areas with bare essential facilities, a checklist will ensure basic standardisation of care in the peri-operative period and improve outcomes.

Adaptation of structured checklists, far from being restrictive, standardises human behaviour, places less reliance on memory, improves consistency and by verbalizing safety brings about a change in the behaviour of a team. They have been proven to reduce communication errors. Whether the improvement is due to "Hawthorne effect" or an actual change in behaviour, only time will tell. What needs also to be remembered is that a checklist is no substitute for personal vigilance. It should not lead to complacency; errors have been known to occur inspite of checklists. In $88 \%$ of cases of retained swabs the swab count at the end of the operation was reported as correct [9].
We cannot forever hide under the guise that clinical decision making, is different, complex and ambiguous. There is need for a change in this culture. For too long, patient safety has taken a backseat. It is time that our policies become patient centred. A pilot, regardless of the number of hours of flying under his belt, unlike a surgeon or a physician, uses a checklist religiously because any error can be fatal for him and his passengers. There is a need to promote effective team working, develop organisational and personal leadership skills by introducing formal training in patient safety procedures during medical school and surgical residency. In this, the Association of surgeons of India needs to take up the lead and be seen to make surgery safe for everyone anywhere in India.

\section{References}

1. Kaul A, McColloch P (2004) A prospective study of patient harm in general surgery. Br J Surg 91(Suppl 1):143

2. Leape LL (1994) Error in medicine. JAMA 272:1851-7

3. Healy MA, Shackford ST, Osler TM, Rogers FB, Burns E (2002) Complications in surgical patients. Arch Surg 137:611-618

4. Mishra A, Giddings AEB (2008) Risk management in surgery. In: Taylor I, Johnson CD (eds) Recent advance in surgery 30. Churchill Livingstone, Edinburgh, pp 1-10

5. Reason J (2000) Human error: models and management. BMJ 320:768-770

6. Helmreich RL, Merritt AC, Wilhelm JA (1999) The evolution of crew resource management training in commercial aviation. Int $\mathrm{J}$ Aviation Psychology 9:19-32

7. Sexton JB, Thomas EJ, Helmreich RL (2000) Error, stress, and teamwork in medicine and aviation: cross sectional surveys. BMJ 320:745-9

8. Haynes AB, Weiser TG, Berry WR, Lipsitz SR, Breizat AH, Dellinger EP et al (2009) Safe surgery saves lives study group. A surgical safety checklist to reduce morbidity and mortality in a global population. N Engl J Med 360:491-9

9. Gawande AA, Studdert DM, Orav EJ, Brennan TA, Zinner MA (2003) Patient safety: risk factors for retained instruments and sponges after surgery. N Engl J Med 348:229-35

10. http://www.who.int/patientsafety/safesurgery/tools_resources/ SSSL checkilist.

11. Lingard L, Regehr G, Orser B, Reznick R, Baker GR, Doran D, Espin S, Bohnen J, Whyte S (2008) Evaluation of a preoperative checklist and team briefing among surgeons, nurses, and anaesthesiologists to reduce failures in communication. Arch Surg $143: 12-17$ 